\title{
Protectin D1 protects against lipopolysaccharide-induced acute lung injury through inhibition of neutrophil infiltration and the formation of neutrophil extracellular traps in lung tissue
}

\author{
ZHIYANG WU ${ }^{1 *}$, LUYAO ZHANG $^{2 *}$, XIANGYANG ZHAO ${ }^{1}$, ZHI LI $^{1}$, HAINING LU ${ }^{1}$, \\ CHANYUAN BU ${ }^{1}$, RUI WANG ${ }^{1}$, XIAOFEI WANG ${ }^{1}$, TIANTIAN CAI ${ }^{1}$ and DAWEI WU ${ }^{1}$ \\ ${ }^{1}$ Department of Critical Care Medicine, Qilu Hospital (Qingdao), Cheeloo College of Medicine, Shandong University, \\ Qingdao, Shandong 266035; ${ }^{2}$ Department of Pathology, School of Medicine and Holistic Integrative Medicine, \\ Nanjing University of Chinese Medicine, Nanjing, Jiangsu 210023, P.R. China
}

Received July 6, 2020; Accepted January 28, 2021

DOI: $10.3892 /$ etm.2021.10508

\begin{abstract}
Protectin D1 (PD1), a DHA-derived lipid mediator, has recently been shown to possess anti-inflammatory and pro-resolving properties. To date, little is known about the effect of PD1 on lipopolysaccharide (LPS)-induced acute lung injury (ALI) in mice. The aim of the present study was to investigate the therapeutic effects of PD1 on LPS-induced ALI and its potential mechanisms of action. ALI was induced via an intraperitoneal injection of LPS, where PD1 (2 ng/mouse) was administered intravenously $30 \mathrm{~min}$ after LPS challenge. Mice were sacrificed $24 \mathrm{~h}$ after modeling. Lung histopathological changes were assessed using hematoxylin and eosin staining and myeloperoxidase (MPO) activity was tested using immunohistochemistry. Tumor necrosis- $\alpha$ and interleukin- 6 levels in the bronchoalveolar lavage fluid (BALF) and serum were measured using ELISA. To detect neutrophil extracellular traps produced by infiltrated neutrophils in the lung tissue, immunofluorescence staining was performed using anti-MPO and anti-histone $\mathrm{H} 3$ antibodies. The results indicated that PD1 significantly attenuated histological damage and neutrophil infiltration in lung tissue, reduced the lung wet/dry weight ratio, protein concentration and proinflammatory cytokine levels in BALF and decreased proinflammatory cytokine levels in serum. Moreover, neutrophil citrullinated histone $\mathrm{H} 3$ (CitH3) expression was also reduced after PD1 administration. In conclusion, PD1 attenuated LPS-induced ALI in mice via
\end{abstract}

Correspondence to: Dr Dawei Wu, Department of Critical Care Medicine, Qilu Hospital (Qingdao), Cheeloo College of Medicine, Shandong University, 758 Hefei Road, Qingdao, Shandong 266035, P.R. China

E-mail: qiluwdw@163.com

*Contributed equally

Key words: protectin D1, acute lung injury, neutrophil infiltration, neutrophil extracellular trap inhibition of neutrophil extracellular trap formation in lung tissue. Therefore, PD1 administration may serve to be a new strategy for treating ALI.

\section{Introduction}

Acute lung injury (ALI) and its most severe form, acute respiratory distress syndrome (ARDS), are widespread inflammatory processes in the lung caused by pneumonia, sepsis, trauma and aspiration. ALI is characterized by pulmonary interstitial edema, deteriorated lung compliance and subsequent severe hypoxemia $(1,2)$. Despite great progress in our understanding of the pathophysiology of the condition, ALI still has a relatively high mortality of $30-40 \%$ in intensive care units of the United States and no specific drug has been approved for clinical treatment. To reduce this mortality rate treatment and prevention strategies for ALI need to be improved (3-5).

Lipopolysaccharide (LPS), a glycolipid of the outer membrane of gram-negative bacteria (6), has been determined to be an important factor in the activation of inflammatory signaling cascades in ALI development (5). Intraperitoneal injection of LPS in mice is an effective method to establish an ALI model.

Protectin D1 (PD1) is a bioactive product generated from docosahexaenoic acid (DHA) and has been reported to exert anti-inflammatory effects in various disorders, including acute kidney injury and neurodegenerative diseases $(7,8)$. A previous study suggested that PD1 could promote the resolution of inflammation in a murine model of LPS-induced ALI (9). However, the underlying molecular mechanism of the anti-inflammatory effect of PD1 in ALI is not well understood.

Neutrophils are part of the host's first line of defense against invading pathogens. Activated neutrophils can release networks of nuclear DNA, enzymes and histones, which are known as neutrophil extracellular traps (NETs) (10). This process, known as NETosis (11), was initially considered to play a host-protective role against infection (12). NETosis is important in the pathophysiology of many conditions, including rheumatoid arthritis (13), small vessel vasculitis (14) and diabetes (15). Recently, NETs formation was identified in 
LPS-induced ALI (16) and further studies revealed that NETs was directly responsible for alveolar damage and epithelial cell death (17). Targeting NET formation in ALI may provide an avenue for treatment that has yet to be explored. However, little is known regarding the beneficial role of PD1 in inflammatory responses and NETs formation in ALI.

In the present study, the significance of PD1 in mouse models of LPS induced ALI was explored and the effect of PD1 on NETs formation evaluated.

\section{Materials and methods}

Animals. A total of 28 male ICR mice (age, 6-8 weeks; weight, 21-28 g) were obtained from Jinan Pengyue Experimental Animal Breeding Co. Ltd. All mice were housed at an ambient temperature of $20-22^{\circ} \mathrm{C}$ with a relative humidity of $5 \pm 5 \%$ and on a 12 -h light-dark cycle in specific pathogen-free (SPF) facilities with free access to food and water. All animal protocols were approved by the Institutional Animal Care and Use Committee of Qilu Hospital (Qingdao; China) and were performed in accordance with the guidelines of the Animal Care and Use Committee (18).

Experimental model and treatments. The mice were randomly divided into four groups (7 mice per group): The control group, control + PD1 group, LPS group and LPS + PD1 group. The LPS model was induced by intraperitoneal injection of $10 \mathrm{mg} / \mathrm{kg}$ LPS, as previous described $(19,20)$. Mice in the control group and the control + PD1 group were administered an equal volume of PBS. At $30 \mathrm{~min}$ after LPS/PBS treatment, PD1 treated mice were injected with PD1 (2 ng/mouse) via the angular vein. All mice were sacrificed $24 \mathrm{~h}$ after the first administration of LPS. All animals were anesthetized with an intraperitoneal administration of sodium pentobarbital $(50 \mathrm{mg} / \mathrm{kg})$ before sacrifice. Euthanasia was performed under anesthesia using cardiac puncture resulting in exsanguination. Death was confirmed by cervical dislocation. Complete cessation of heartbeat and breathing and disappearance of reflexes were used as indicators to judge the death of mice. Lung tissues and blood samples were obtained for further analyzes.

Histological analysis. The inferior lobe of the right lung was fixed for $24 \mathrm{~h}$ at room temperature with $4 \%$ formaldehyde, embedded in paraffin and sectioned at a thickness of $5-\mu \mathrm{m}$. The sections were stained with hematoxylin at $25^{\circ} \mathrm{C}$ for $10 \mathrm{~min}$ and eosin (H\&E) at $25^{\circ} \mathrm{C}$ for $3 \mathrm{~min}$. Histopathological scoring analysis of the lung tissues was evaluated by TC and $\mathrm{XW}$ who were blinded to the group information, with a light microscope at magnifications of $x 400$ according to the criteria previously described (21). Briefly, 24 areas in the lung parenchyma were graded on a scale of 0-4 (0, absent and appears normal; 1 , light; 2 , moderate; 3 , strong; 4 , intense) for congestion, edema, infiltration of inflammatory cells and hemorrhaging.

Lung wet-to-dry weight ratio. The superior lobe of the right lung was removed and weighed to measure the wet weight. The lungs were then dried in an oven at $60^{\circ} \mathrm{C}$ for 3 days to obtain the dry lung weight.
Pulmonary wet/dry weight ratios were calculated as follows: Wet/dry weight ratio=(wet lung weight-dry lung weight)/dry lung weight.

Measurement of protein concentrations and cytokines in bronchoalveolar lavage fluid $(B A L F)$. The left lungs were rinsed with $1 \mathrm{ml}$ of PBS through the tracheal cannula three times to obtain BALF. The BALF was centrifuged at $850 \mathrm{xg}$ for $10 \mathrm{~min}$ at $4^{\circ} \mathrm{C}$. The supernatant was collected for protein measurement using a bicinchoninic acid protein assay kit (Thermo Fisher Scientific, Inc.) according to the manufacturer's protocols. Tumor necrosis factor $(\mathrm{TNF}-\alpha)$ and interleukin- 6 (IL-6) levels in BALF were measured using TNF- $\alpha$ ELISA kit [cat. no. EK282/3; MultiSciences (Lianke) Biotech. Co., Ltd.] and IL-6 ELISA kit [cat. no. EK206/3; MultiSciences (Lianke) Biotech. Co., Ltd.].

Measurement of serum TNF- $\alpha$ and IL-6. The serum levels of TNF- $\alpha$ and IL- 6 were determined using TNF- $\alpha$ ELISA kit [cat. no. EK282/3; MultiSciences (Lianke) Biotech. Co., Ltd.] and IL-6 ELISA kit [cat. no. EK206/3; MultiSciences (Lianke) Biotech. Co., Ltd.] according to the manufacturer's instructions.

Immunohistochemical analysis of myeloperoxidase (MPO). Immunohistochemical analysis of MPO was performed according to previously described methods $(22,23)$. One portion of the lungs was fixed overnight at $4^{\circ} \mathrm{C}$ in freshly prepared formaldehyde (Sigma-Aldrich; Merck KGaA) in PBS, pH 7.4. The tissues were then embedded in paraffin. Lung tissue sections (at a thickness of $5 \mu \mathrm{m}$ ) were deparaffinized in xylene at room temperature for $15 \mathrm{~min}$, rehydrated using a descending ethanol gradient and heated in $10 \mathrm{mM}$ sodium citrate buffer ( $\mathrm{pH} \mathrm{6.0)}$ ) at $95^{\circ} \mathrm{C}$ for $5 \mathrm{~min}$ in a microwave oven for antigen retrieval. Endogenous peroxidase activity was blocked by immersing the slides in $3 \%$ hydrogen peroxide at $15-25^{\circ} \mathrm{C}$ for $10 \mathrm{~min}$. After antigen retrieval, the tissue sections were immersed in 5\% BSA (Sigma-Aldrich; Merck KGaA) for $1 \mathrm{~h}$ at room temperature. The tissue sections were then incubated with a primary antibody against MPO (1:100 dilution; cat. no. ab9535; Abcam, Inc.) at $4^{\circ} \mathrm{C}$ overnight. The tissue sections were then rinsed in TBS and incubated with a horseradish peroxidase (HRP)-conjugated secondary anti-rabbit antibody (1:1,000 dilution; cat. no. ab97080; Abcam) for $1 \mathrm{~h}$ at room temperature. The tissue sections were again washed in TBS and the bound peroxidase was detected by incubating the tissue sections in 3,3'-diaminobenzidine substrate at room temperature for $3 \mathrm{~min}$. The tissue sections were then washed in distilled water and the nuclei were counterstained with Mayer's hematoxylin for $1 \mathrm{~min}$ at room temperature, followed by two rinses in distilled water. The sections were dehydrated by serial immersion, for $1 \mathrm{~min}$ each, in $70 \%$ ethanol and $95 \%$ ethanol, followed by 2 min each in two changes of $100 \%$ ethanol and two changes of xylene. A coverslip was mounted over each tissue section using Pertex ${ }^{\circledR}$ mounting medium (Pioneer Research Chemical, Ltd.). Imaging was performed using a fluorescence microscope (Nikon MODEL ECLIPSE Ci-L microscope; Nikon Corporation) at magnifications of X100 and 400 . 
A

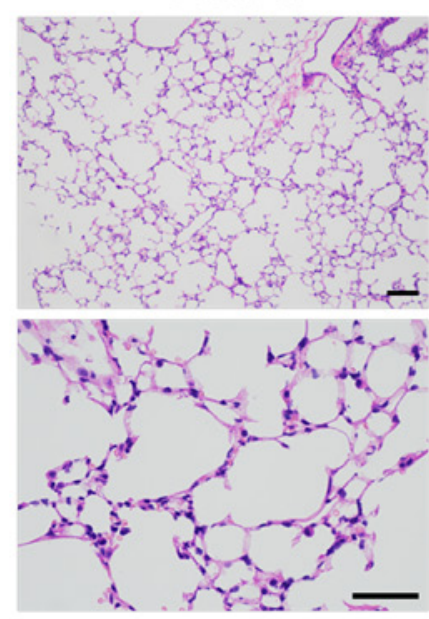

B

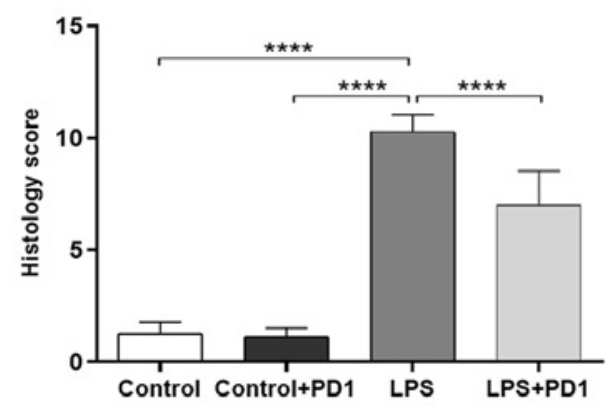

Control+PD1

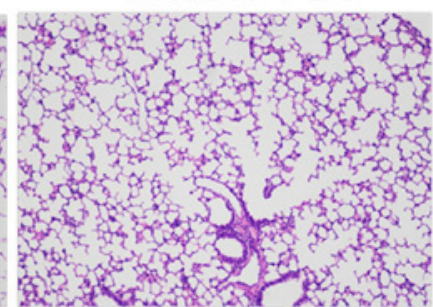

LPS

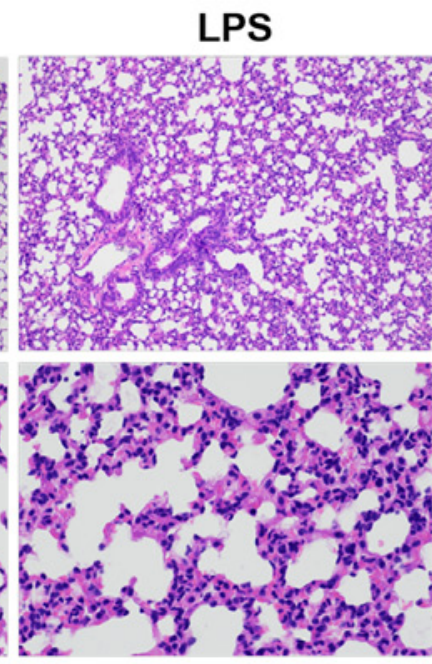

C
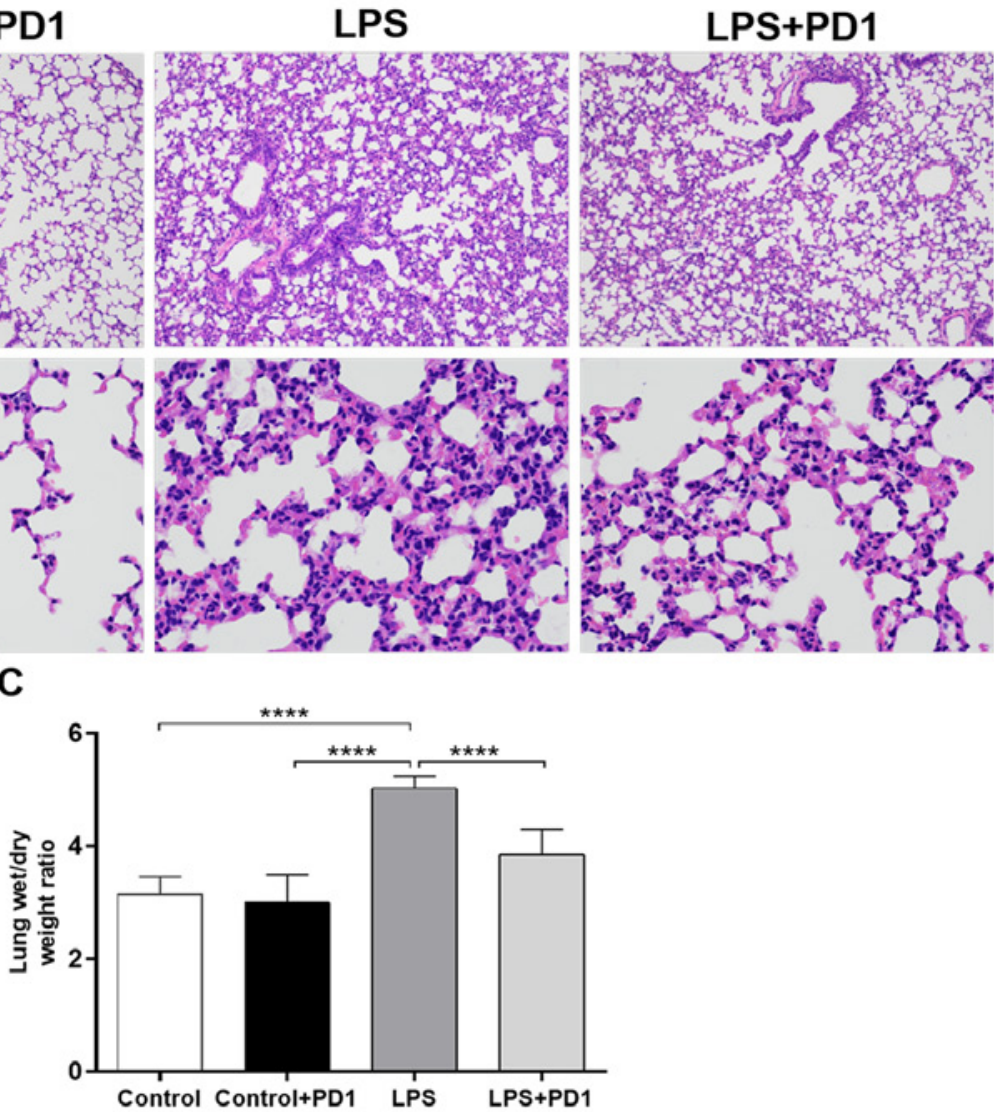

Figure 1. PD1 attenuates LPS-induced lung histopathological changes and pulmonary edema. (A) Representative H\&E staining of lung tissues. Scale bars: $100 \mu \mathrm{m}$. (B) Histopathological scores of lung tissues. (C) Wet/dry weight ratios of lung tissues. $\mathrm{n}=7$ in each group. PD1, protectin D1; LPS, lipopolysaccharide. ${ }^{* * * * *} \mathrm{P}<0.0001$

Immunofluorescence. To detect NET produced by infiltrated neutrophils in the lung tissue, immunofluorescent staining was performed using anti-MPO and anti-histone $\mathrm{H} 3$ antibodies. Lung tissues were fixed overnight at $4^{\circ} \mathrm{C}$ in freshly prepared formaldehyde (Sigma-Aldrich; Merck KGaA) in PBS, pH 7.4. The tissues were then embedded in paraffin. Lung tissue sections (at a thickness of $6 \mu \mathrm{m}$ ) were deparaffinized and rehydrated in descending concentrations of ethanol. Antigen retrieval was achieved by treating sections with $10 \mathrm{mM}$ citrate buffer and heating in a microwave oven for $15 \mathrm{~min}$ at $95^{\circ} \mathrm{C}$. Samples were then blocked with $20 \%$ normal donkey serum (cat. no. ab7475; Abcam) overnight at $4^{\circ} \mathrm{C}$. After a thorough wash in $0.05 \%$ PBS-Tween-20, the sections were sequentially incubated with rabbit anti-MPO antibody (1:100 dilution; cat. no. ab9535; Abcam) and mouse anti-histone $\mathrm{H} 3$ antibody (1:200 dilution; cat. no. ab1220; Abcam) in a humidified chamber at $4^{\circ} \mathrm{C}$ overnight. After being washed again, the samples were incubated with secondary antibodies that recognize citrullinated histone 3 (CitH3; donkey anti-mouse IgG; Alexa Fluor ${ }^{\circledR} 488$-conjugated; 1:500 dilution; cat. no. ab150105; Abcam) and MPO (donkey anti-rabbit IgG; Alexa Fluor ${ }^{\circledR}$ 647-conjugated; 1:500 dilution; cat. no. ab150075; Abcam) for $1 \mathrm{~h}$ at room temperature. Following another wash, the sections were incubated with 4',6-diamidino-2-phenylindole and dihydrochloride (DAPI; Wuhan Servicebio Technology Co. Ltd.) solution at room temperature for $20 \mathrm{~min}$ and then mounted with anti-fade reagent $(24,25)$. Immunofluorescence images were obtained with a slide scanner (Panoramic MIDI; 3DHISTECH Ltd.) at magnifications of $\mathrm{x} 100$ and 400 and analyzed with ImageJ v1.8.0 software (National Institutes of Health).

Statistical analysis. Statistical analysis was performed using GraphPad Prism 6 software (GraphPad Software Inc.), and the data are presented as the mean \pm standard error (SE). Statistical significance was assessed using one-way ANOVA followed by Tukey's post hoc test. A value of $\mathrm{P}<0.05$ was considered statistically significant.

\section{Results}

PD1 attenuates LPS-induced lung histopathological changes and pulmonary edema. Histopathological analysis was used to determine the protective effects of PD1 on LPS-induced ALI. As indicated by Fig. 1A, lungs in the LPS group showed extensive histopathological changes, including alveolar wall thickening, interstitial edema, and pulmonary congestion, compared to those of control group. PD1 attenuated the severity of lung injuries induced by LPS challenge. As suggested in Fig. 1B, lung injury scores decreased significantly after PD1 administration. The wet/dry weight ratio of the lung was examined to estimate lung edema. As demonstrated in Fig. 1C, the lung wet/dry weight ratio in the LPS group was significantly increased compared with that in the control group. However, this increase was significantly reduced by PD1 treatment. 
A

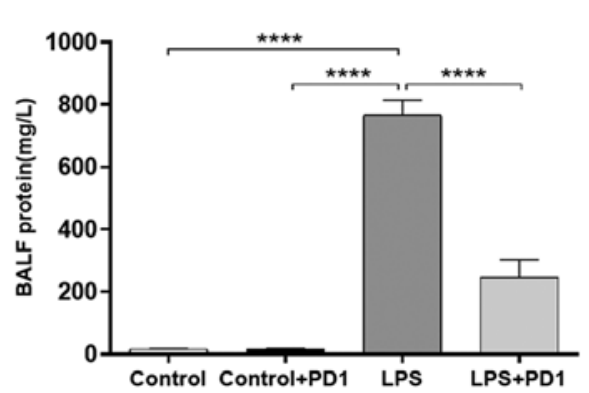

B

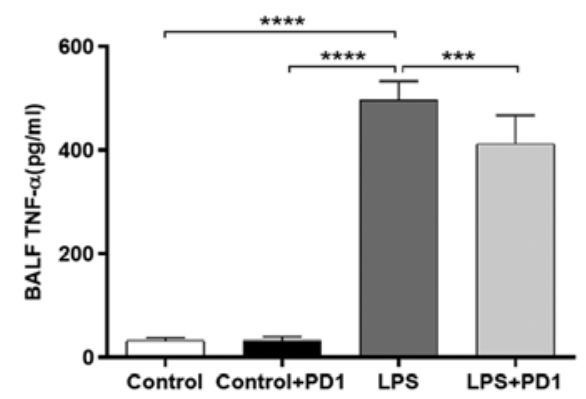

C

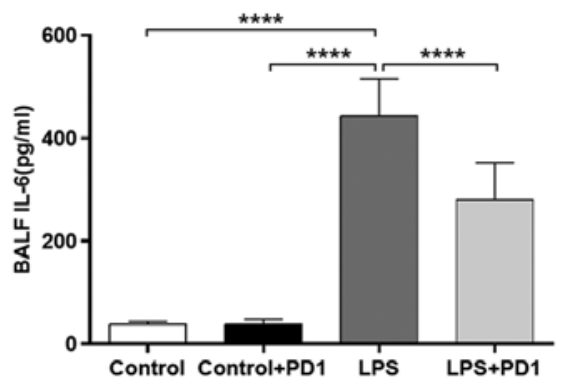

Figure 2. PD1 alleviates protein leakage and reduces proinflammatory cytokine levels in BALF. (A) Protein concentration in BALF. (B) Concentration of TNF- $\alpha$ in BALF. (C) Concentration of IL-6 in BALF. $n=7$ in each group. PD1, protectin D1; LPS, lipopolysaccharide; BALF, bronchoalveolar lavage fluid; TNF- $\alpha$, tumor necrosis factor- $\alpha$; IL-6, interleukin $6 .{ }^{* * * *} \mathrm{P}<0.001$ and ${ }^{* * * * *} \mathrm{P}<0.0001$.

A

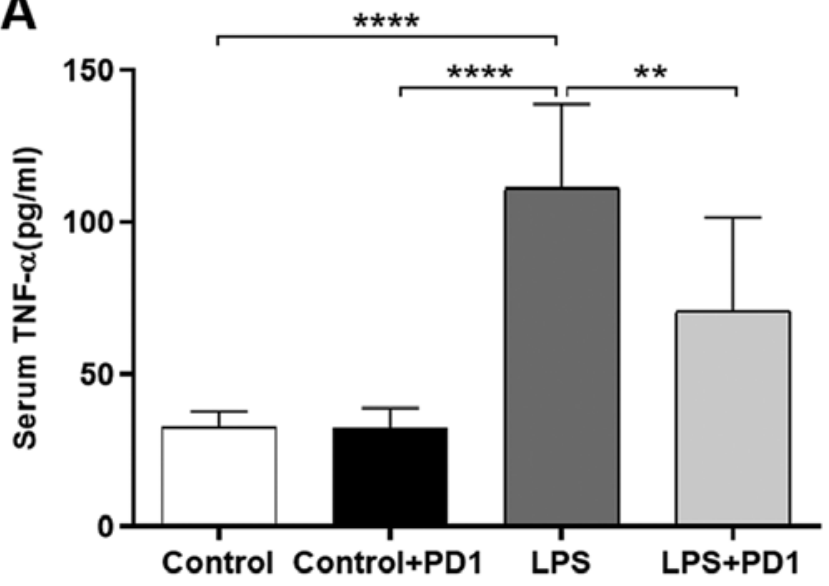

B

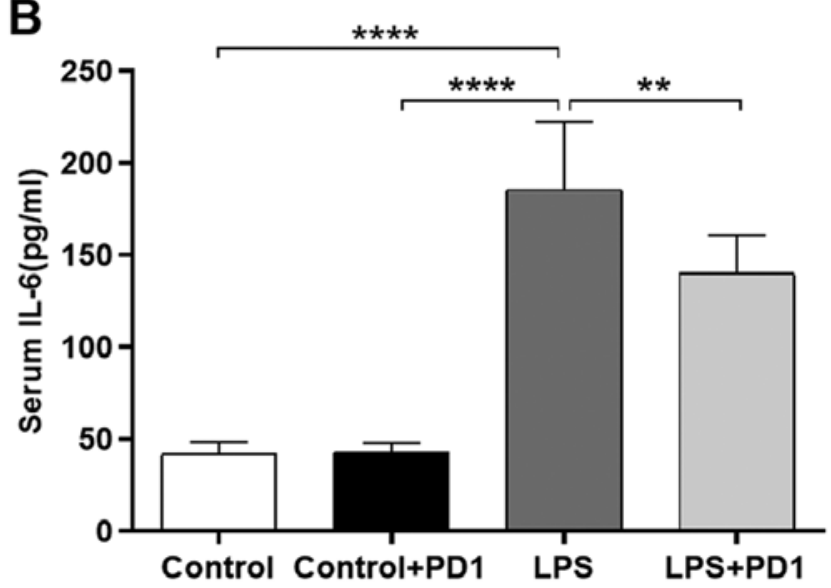

Figure 3. PD1 reduces serum proinflammatory cytokine levels. (A) Serum concentration of TNF- $\alpha$. (B) Serum concentration of IL-6. $\mathrm{n}=7$ in each group. PD1, protectin D1; LPS, lipopolysaccharide; TNF- $\alpha$, tumor necrosis factor- $\alpha$; IL-6, interleukin $6 .{ }^{* *} \mathrm{P}<0.05$ and ${ }^{* * * *} \mathrm{P}<0.001$.

PDI alleviates protein leakage and reduces proinflammatory cytokine levels in BALF. As demonstrated in Fig. 2A, the protein concentration in BALF was increased by LPS challenge in comparison with control while PD1 administration alleviated protein leakage in LPS mice. Proinflammatory cytokines play an important role in the pathogenesis of ALI $(26,27)$. In the present study, the concentrations of TNF- $\alpha$ (Fig. 2B) and IL-6 (Fig. 2C) in BALF were significantly increased in LPS-treated mice compared with control mice. Administration of PD1 decreased LPS-induced inflammatory cytokine (TNF- $\alpha$ and IL-6) production.

PDI reduces serum proinflammatory cytokine levels. As indicated in Fig. 3, the concentrations of TNF- $\alpha$ and IL-6 in the serum were significantly increased in the LPS group compared with the control group. In contrast to that of the LPS group, treatment with PD1 significantly reduced TNF- $\alpha$ and IL-6 release into the serum.

PDI reduces neutrophil infiltration into lung tissue. To investigate the infiltration and accumulation of neutrophils in LPS-induced ALI, immunohistochemistry was carried out on lung sections using an MPO antibody. The expression of MPO (Fig. 4A and B) in the lung tissues of the LPS group was significantly increased compared with that of the control group. PD1 administration significantly reduced the LPS-induced MPO increase.

PD1 significantly reduces NETs formation in mouse lungs. $\mathrm{CitH} 3$ is found in the extracellular space of neutrophils along with DNA as components of NETs. CitH3 is widely accepted as a marker of NETs formation $(28,29)$. NETs were identified on the basis of co-localization of CitH3 with MPO using immunofluorescence. As demonstrated in Fig. 5, neutrophil infiltration, as assessed by MPO and $\mathrm{CitH} 3$ colocalization, was significantly increased in the LPS group in comparison with the control group. Treatment with PD1 reduced neutrophil infiltration and NETs formation in lung tissue of LPS mice, as demonstrated by reduced CitH3/MPO co-localization.

\section{Discussion}

In the present study, the protective effect of PD1 on LPS-induced ALI in mice was evaluated. The data indicated that PD1 significantly attenuated LPS-induced histopathological changes and pulmonary edema and alleviated protein leakage in BALF in comparison with an LPS control. Moreover, administration of PD1 reduced the BALF and serum levels of proinflammatory 
A

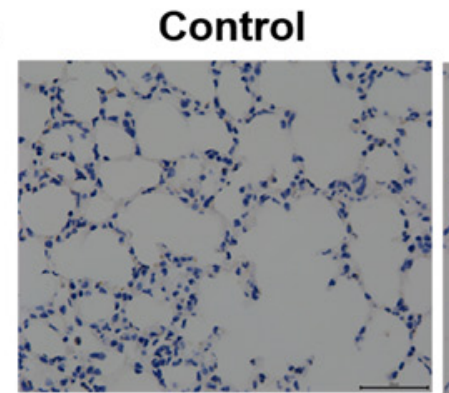

Control+PD1

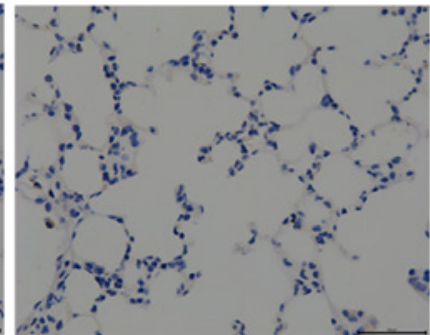

LPS

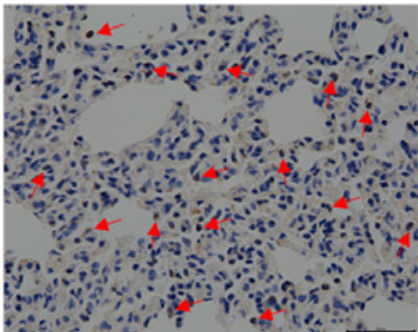

\section{LPS+PD1}

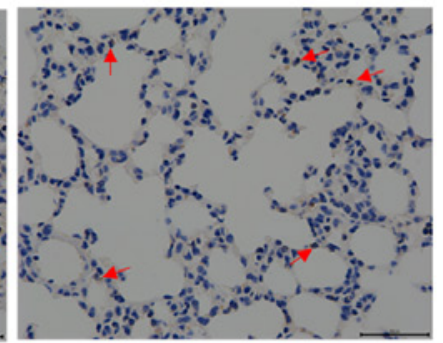

B

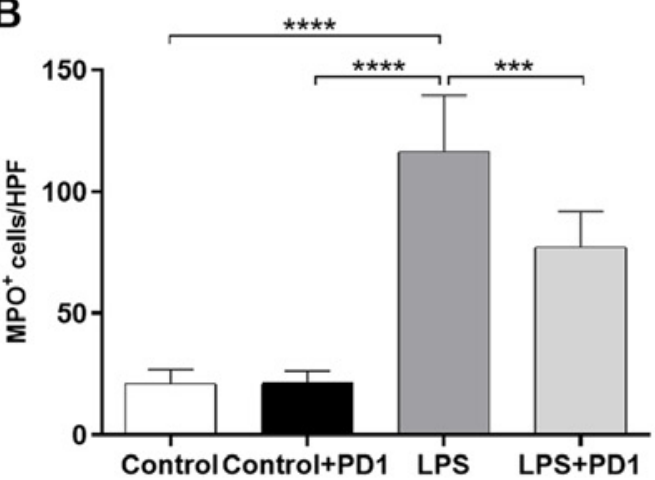

Figure 4. PD1 reduces neutrophil infiltration in lung tissue. (A) Representative immunohistochemical images of MPO expression in the lung tissues. Red arrows represent infiltrating neutrophils. Scale bars: $50 \mu \mathrm{m}$. (B) Quantitative summary of MPO expression. $\mathrm{n}=7$ each group. PD1, protectin D1; LPS, lipopolysaccharide; MPO, myeloperoxidase. ${ }^{* * *} \mathrm{P}<0.001$ and ${ }^{* * * *} \mathrm{P}<0.0001$.

A

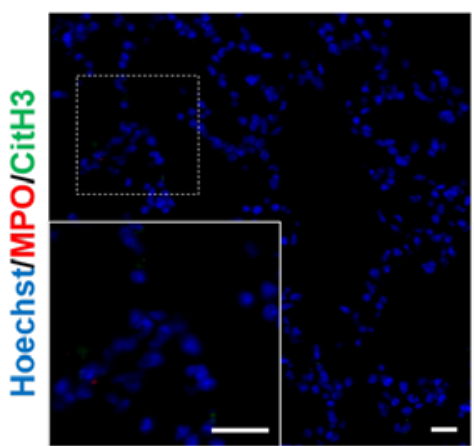

Control

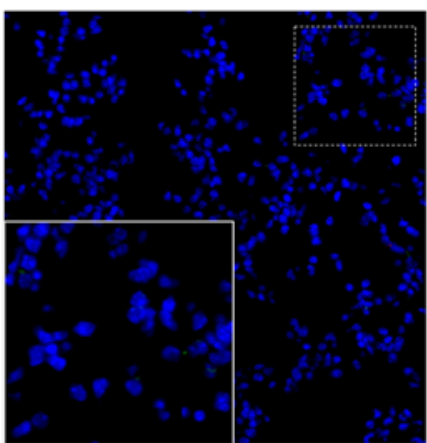

B

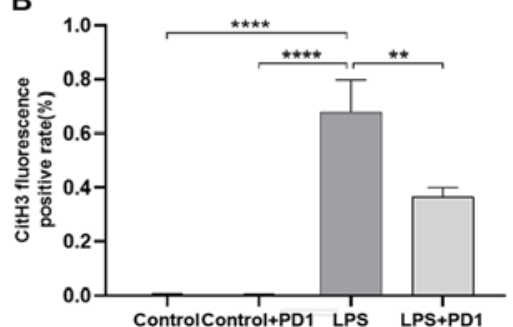

LPS
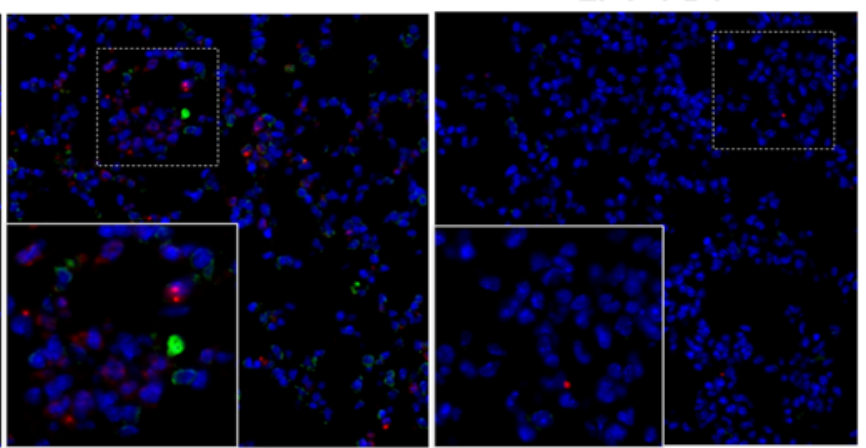

Figure 5. PD1 reduces neutrophil extracellular trap formation in mouse lungs. (A) Colocalization of CitH3 (green) with neutrophils (MPO, red) in the lung tissues. Scale bars: $100 \mu \mathrm{m}$. (B) Quantitative summary of neutrophil CitH3 expression. n=7 each group. PD1, protectin D1; LPS, lipopolysaccharide; CitH3, citrullinated histone 3; MPO, myeloperoxidase. ${ }^{* *} \mathrm{P}<0.01$ and ${ }^{* * * *} \mathrm{P}<0.001$.

cytokines TNF- $\alpha$ and IL-6, reduced the infiltration of inflammatory cells and suppressed the formation of NETs in LPS-induced lung tissue.

ALI and ARDS, the major causes of respiratory failure in intensive care units worldwide, are characterized by uncontrolled inflammatory responses (30). Currently, clinical treatment is limited to supportive care. Novel therapeutic methods or preventive strategies are urgently needed (31). PD1, a bioactive product generated from docosahexaenoic acid (DHA), has been reported to exert anti-inflammatory effects in a murine model of LPS-induced ALI (9). In the present study, the protective effect of PD1 on LPS-induced ALI was further explored. The present data revealed that intraperitoneal injection of LPS resulted in the destruction of alveolar 
structures and pulmonary edema and that PD1 exhibited substantial protective effects against LPS-induced ALI, as evidenced by alleviated pathological injuries and pulmonary edema. However, the underlying molecular mechanism of the anti-inflammatory effect of PD1 on LPS-induced ALI is still not well understood.

A key feature of LPS-induced ALI is an early, robust neutrophilic response. Infiltration of neutrophils and release of proinflammatory cytokines play important roles in ALI (32). A previous study suggested that LPS may promote the production of inflammatory cytokines, which may lead to neutrophil infiltration into lung tissues and initiate focal inflammatory responses $(33,34)$. Excessive accumulation of neutrophils contributes to disturbances in the alveolar-capillary barrier, resulting in the efflux of protein-rich fluid and ultimately pulmonary edema (35). In the present study, the concentrations of protein, TNF- $\alpha$ and IL-6 in BALF were significantly increased in LPS-treated mice compared with control mice. Furthermore, this increase was significantly suppressed by PD1 treatment. Consistent with the BALF results, serum TNF- $\alpha$ and IL- 6 levels in the LPS group were increased significantly compared with those of the control group and were significantly reduced by PD1 administration.

It is well known that neutrophils are crucial in the initiation and exacerbation of pathological processes and inflammatory responses (32). Inhibition of neutrophil recruitment has been demonstrated to attenuate LPS-induced ALI by reducing the production of proinflammatory cytokines (36). In the present study, administration of PD1 significantly inhibited neutrophil infiltration and accumulation in the lungs.

NETs have been identified as critical mediators of pulmonary vascular dysfunction and the pathogenesis of lethal endotoxemia $(29,37)$. Inhibition of NET formation was demonstrated to alleviate LPS-induced pulmonary dysfunction and improve survival in a mouse model of lethal endotoxemia (29). Targeted intervention in NETs formation may be a new strategy for the clinical treatment of AP. In the present study, CitH3 released from infiltrating neutrophils was measured to assess NETs formation in LPS-induced lung tissue. CitH3 expression was colocalized with MPO, suggesting that $\mathrm{CitH} 3$ was released from infiltrating neutrophils. PD1 administration significantly reduced neutrophil infiltration and NETs formation in LPS-induced lung tissue.

In conclusion, the present study demonstrated that PD1 protects against LPS-induced ALI by inhibiting the infiltration of neutrophils and the formation of NETs in lung tissue. PD1 may be a promising therapeutic candidate for the clinical treatment of ALI.

\section{Acknowledgements}

Not applicable.

\section{Funding}

The present study was supported by grants from the Scientific Research Foundation of Qilu Hospital (grant no. QDKY2019RX11) and The Youth Program of the National Natural Science Foundation of China (grant no. 81704164).

\section{Availability of data and materials}

The datasets used and/or analyzed during the current study are available from the corresponding author on reasonable request.

\section{Authors' contributions}

ZW and DW designed the study. LZ, HL and ZW performed the experiments and collected the data. XZ, ZL and $\mathrm{CB}$ contributed to the interpretation of data for the work. RW, TC and XW analyzed the data, and ZW and LZ prepared the manuscript and assessed the authenticity of all the raw data. All authors read and approved the final manuscript.

\section{Ethics approval and consent to participate}

All animal protocols were approved by the Institutional Animal Care and Use Committee of Qilu Hospital (Qingdao), Shandong University (Qingdao, China).

\section{Patient consent for publication}

Not applicable.

\section{Competing interests}

The authors declare that they have no competing interests.

\section{References}

1. Ferguson ND, Frutos-Vivar F, Esteban A,Fernández-Segoviano P, Aramburu JA, Nájera L and Stewart TE: Acute respiratory distress syndrome: Underrecognition by clinicians and diagnostic accuracy of three clinical definitions. Crit Care Med 33: 2228-2234, 2005.

2. Mokra D and Kosutova P: Biomarkers in acute lung injury. Respir Physiol Neurobiol 209: 52-58, 2015.

3. Matthay MA and Zemans RL: The acute respiratory distress syndrome: Pathogenesis and treatment. Annu Rev Pathol 6: 147-163, 2011.

4. Goss CH, Brower RG, Hudson LD and Rubenfeld GD: Incidence of acute lung injury in the United States. Crit Care Med 31: 1607-1611, 2003.

5. Rubenfeld GD, Caldwell E, Peabody E, Weaver J, Martin DP, Neff M, Stern EJ and Hudson LD: Incidence and outcomes of acute lung injury. N Engl J Med 353: 1685-1693, 2005.

6. Rossol M, Heine H, Meusch U, Quandt D, Klein C, Sweet MJ and Hauschildt S: LPS-induced cytokine production in human monocytes and macrophages. Crit Rev Immunol 31: 379-446, 2011.

7. Xu ZZ, Liu XJ, Berta T, Park CK, Lü N, Serhan CN and Ji RR: Neuroprotectin/protectin D1 protects against neuropathic pain in mice after nerve trauma. Ann Neurol 74: 490-495, 2013.

8. Duffield JS, Hong S, Vaidya VS, Lu Y, Fredman G, Serhan CN and Bonventre JV: Resolvin D series and protectin D1 mitigate acute kidney injury. J Immunol 177: 5902-5911, 2006.

9. Li X, Li C, Liang W, Bi Y, Chen M and Dong S: Protectin D1 promotes resolution of inflammation in a murine model of lipopolysaccharide-induced acute lung injury via enhancing neutrophil apoptosis. Chinese Med J (Engl) 127: 810-814, 2014.

10. Brinkmann V, Reichard U, Goosmann C, Fauler B, Uhlemann Y, Weiss DS, Weinrauch Y and Zychlinsky A: Neutrophil extracellular traps kill bacteria. Science 303: 1532-1535, 2004.

11. Kaplan MJ and Radic M: Neutrophil extracellular traps: Double-edged swords of innate immunity. J Immunol 189: 2689-2695, 2012.

12. Fuchs TA, Abed U, Goosmann C, Hurwitz R, Schulze I, Wahn V, Weinrauch Y, Brinkmann V and Zychlinsky A: Novel cell death program leads to neutrophil extracellular traps. J Cell Biol 176: 231-241, 2007. 
13. Sur Chowdhury C, Giaglis S, Walker UA, Buser A, Hahn S and Hasler P: Enhanced neutrophil extracellular trap generation in rheumatoid arthritis: Analysis of underlying signal transduction pathways and potential diagnostic utility. Arthritis Res Ther 16: R122, 2014.

14. Kessenbrock K, Krumbholz M, Schönermarck U, Back W, Gross WL, Werb Z, Gröne HJ, Brinkmann V and Jenne DE: Netting neutrophils in autoimmune small-vessel vasculitis. Nat Med 15: 623-625, 2009.

15. Wong SL, Demers M, Martinod K, Gallant M, Wang Y, Goldfine AB, Kahn CR and Wagner DD: Diabetes primes neutrophils to undergo NETosis, which impairs wound healing. Nat Med 21: 815-819, 2015.

16. Li H, Zhou X, Tan H, Hu Y, Zhang L, Liu S, Dai M, Li Y, Li Q, Mao Z, et al: Neutrophil extracellular traps contribute to the pathogenesis of acid-aspiration-induced ALI/ARDS Oncotarget 9: 1772-1784, 2018.

17. Saffarzadeh M, Juenemann C, Queisser MA, Lochnit G, Barreto G, Galuska SP, Lohmeyer J and Preissner KT: Neutrophil extracellular traps directly induce epithelial and endothelial cell death: A predominant role of histones. PLoS One 7: e32366, 2012.

18. Kertz AF: Animal care and use: An issue now and in the future. J Anim Sci 74: 257-261, 1996.

19. Yu D, Fang X, Xu Y, Xiao H, Huang T, Zhang Y, Ge Y, Li Y, Zong L and Gao J: Rev-erb $\alpha$ can regulate the NF- $\kappa$ B/NALP3 pathway to modulate lipopolysaccharide-induced acute lung injury and inflammation. Int Immunopharmacol 73: 312-320, 2019.

20. Xiao Z, Jia B, Zhao X, Bi S and Meng W: Attenuation of lipopolysaccharide-induced acute lung injury by Cyclosporine-A via suppression of mitochondrial DNA. Med Sci Monit 24: 7682-7688, 2018.

21. Hagiwara S, Iwasaka H, Maeda $\mathrm{H}$ and Noguchi T: Landiolol, an ultrashort-acting beta1-adrenoceptor antagonist, has protective effects in an LPS-induced systemic inflammation model. Shock 31: 515-520, 2009.

22. Zhai KF, Duan H, Cui CY, Cao YY, Si JL, Yang HJ, Wang YC, Cao WG, Gao GZ and Wei ZJ: Liquiritin from glycyrrhiza uralensis attenuating rheumatoid arthritis via reducing inflammation, suppressing angiogenesis, and inhibiting MAPK signaling pathway. J Agric Food Chem 67: 2856-2864, 2019.

23. Zhai KF, Duan H, Chen Y, Khan GJ, Cao WG, Gao GZ, Shan LL and Wei ZJ: Apoptosis effects of imperatorin on synoviocytes in rheumatoid arthritis through mitochondrial/caspase-mediated pathways. Food Funct 9: 2070-2079, 2018.

24. Zhai KF, Duan H, Khan GJ, Xu H, Han FK, Cao WG, Gao GZ, Shan LL and Wei ZJ: Salicin from alangium Chinense ameliorates rheumatoid arthritis by modulating the Nrf2-HO-1-ROS pathways. J Agric Food Chem 66: 6073-6082, 2018.
25. Zhai KF, Zheng JR, Tang YM, Li F, Lv YN, Zhang YY, Gao Z, Qi J, Yu BY and Kou JP: The saponin D39 blocks dissociation of non-muscular myosin heavy chain IIA from TNF receptor 2, suppressing tissue factor expression and venous thrombosis. Br J Pharmacol 174: 2818-2831, 2017.

26. Kurundkar D, Kurundkar AR, Bone NB, Becker EJ Jr, Liu W, Chacko B, Darley-Usmar V, Zmijewski JW and Thannickal VJ: SIRT3 diminishes inflammation and mitigates endotoxin-induced acute lung injury. JCI insight 4: e120722, 2019.

27. Dolinay T, Kim YS, Howrylak J, Hunninghake GM, An $\mathrm{CH}$, Fredenburgh L, Massaro AF, Rogers A, Gazourian L, Nakahira K, et al: Inflammasome-regulated cytokines are critical mediators of acute lung injury. Am J Respir Crit Care Med 185: 1225-1234, 2012.

28. Deng Q, Pan B, Alam HB, Liang Y, Wu Z, Liu B, Mor-Vaknin N, Duan X, Williams AM, Tian Y, et al: Citrullinated histone $\mathrm{H} 3$ as a therapeutic target for endotoxic shock in mice. Front Immunol 10: 2957, 2019.

29. Liang Y, Pan B, Alam HB, Deng Q, Wang Y, Chen E, Liu B, Tian Y, Williams AM, Duan X, et al: Inhibition of peptidylarginine deiminase alleviates LPS-induced pulmonary dysfunction and improves survival in a mouse model of lethal endotoxemia. Eur J Pharmacol 833: 432-440, 2018.

30. Jiang W, Luo F, Lu Q, Liu J, Li P, Wang X, Fu Y, Hao K, Yan T and Ding X: The protective effect of Trillin LPS-induced acute lung injury by the regulations of inflammation and oxidative state. Chem Biol Interact 243: 127-134, 2016.

31. Patel VJ, Biswas Roy S, Mehta HJ, Joo M and Sadikot RT: Alternative and natural therapies for acute lung injury and acute respiratory distress syndrome. Biomed Res Int 2018: 2476824 , 2018.

32. Grommes $\mathbf{J}$ and Soehnlein O: Contribution of neutrophils to acute lung injury. Mol Med 17: 293-307, 2011.

33. Strieter RM, Belperio JA and Keane MP: Cytokines in innate host defense in the lung. J Clin Invest 109: 699-705, 2002.

34. Jiang C, Ting AT and Seed B: PPAR-gamma agonists inhibit production of monocyte inflammatory cytokines. Nature 391: 82-86, 1998.

35. Pugin J, Verghese G, Widmer MC and Matthay MA: The alveolar space is the site of intense inflammatory and profibrotic reactions in the early phase of acute respiratory distress syndrome. Crit Care Med 27: 304-312, 1999.

36. Lee JM, Yeo CD, Lee HY, Rhee CK, Kim IK, Lee DG, Lee SH and Kim JW: Inhibition of neutrophil elastase contributes to attenuation of lipopolysaccharide-induced acute lung injury during neutropenia recovery in mice. J Anesth 31: 397-404, 2017.

37. Liu S, Su X, Pan P, Zhang L, Hu Y, Tan H, Wu D, Liu B, Li H, $\mathrm{Li} \mathrm{H}$, et al: Neutrophil extracellular traps are indirectly triggered by lipopolysaccharide and contribute to acute lung injury. Sci Rep 6: 37252, 2016. 